\title{
UM ENTENDIMENTO LINEAR SOBRE A TEORIA DE PEPLAU E OS PRINCÍPIOS DA REFORMA PSIQUIÁTRICA BRASILEIRA
}

An Agreement About the Peplau's Theory and the

Principles of the Brazilian Psychiatric Reformation

Un Entendimiento Sobre la Teoría de Peplau y

Los Principios de la Reforma Psiquiátrica Brasileña

Taís Veronica Macedo Cardoso

Rosane Mara Pontes de Oliveira

Cristina Maria Douat Loyola

\begin{abstract}
Resumo
Trata de uma análise sobre os conceitos contidos na teoria de Hildegard Peplau, datados de 1952 e associá-los aos preceitos da Reforma Psiquiátrica Brasileira considerando a realidade social e histórica dessa associação. Partiu-se da análise sistemática do livro Interpersonal Relations in Nursing e dos conceitos nele existentes. 0 estudo foi realizado sob o enfoque da metanálise, que ajudou na compreensão dos conceitos estudados. A partir da análise das duas referências teóricas, compreendemos que os conceitos chaves de Hildegard Peplau ainda hoje são aplicáveis e capazes de orientar com excelência para um cuidado de enfermagem psiquiátrica que atenda aos preceitos organizadores da Reforma Psiquiátrica Brasileira.
\end{abstract}

Palavras-chave: Enfermagem Psiquiátrica. Relacionamento Interpessoal. Reforma Psiquiátrica.

\begin{abstract}
The object of study to be developed deals with the concept contained in the theory of psychiatric nursing proposed by Hildegard Peplau and to associate them with the rules of the Brazilian Psychiatric Reformation. Although the beginning was to apprehend the concepts of the theory of psychiatric nursing using the systematic analysis considered in the "Interpersonal Relations". The study was carried through under the approach of metanalise, that in it helped us to understand the studied concepts. From it analyzes of the data, we understand that still, allowing the agreement of the importance of the transformations of this practice, providing an improvement of the helping practice and initiating assistance based on the models of psychosocial rehabilitation.
\end{abstract}

Keywords: Psychiatric Nursing. Interpersonal Relationship. Psychiatric Reformation.

\section{Resumen}

Trata del análisis de los conceptos contenidos en la teoria de Hildegard Peplau datado de 1952 y de la asociación a los preceptos de la Reforma Psiquiátrica Brasileña considerando la realidad social e histórica de esta asociación. Se partió del análisis sistemático del libro "Interpersonal Relations in Nursing" y de sus conceptos. El estudio fue realizado bajo el enfoque del metanalisis, que nos ayudó a comprender los conceptos estudiados. A partir del análisis de las teorias, comprendemos que los conceptos claves de Hildegard Peplau aún son capaces de orientar com excelencia en los dias actuales un cuidado de enfermería psiquiátrica que atienda a los preceptos organizadores de la Reforma Psiquiátrica Brasileña.
Palabras clave: Enfermería Psiquiátrica. Relacionamiento Interpersonal. Reforma Psiquiátrica. 


\section{INTRODUÇÃO}

A assistência psiquiátrica brasileira vem passando, desde o final da década de 60 , por um movimento de reestruturação de saberes e conseqüentemente de suas práticas conseqüentes. Reformar para a psiquiatria brasileira é caracterizado por duas necessidades: mudança de olhar e tomada de consciência ${ }^{1,2}$.

Esse movimento pode ser entendido como um conjunto de estratégias que visam reordenar as práticas de cuidado relacionadas à Saúde Mental, dando conta não somente de uma questão empírica, que se guia pela experiência da prática, mas, acima de tudo, de uma nova forma de pensar a "loucura".

Falamos de teorias, propostas de transformação compatíveis com essa perspectiva de mudança. 0 enfermeiro tem seu histórico de cuidados, na saúde mental e psiquiatria, pautado no modelo hospitalocêntrico, embora a assistência de enfermagem psiquiátrica tenha conceitos propostos por teóricas como Hildegard Peplau, que transcendem espaços físicos, hospitalares ou extra-hospitalares. A prática, no entanto, ainda é determinada pelo cenário manicomial. Para a enfermagem psiquiátrica brasileira, o desafio está em contemplar esses princípios fundamentais que respaldam sua existência, dentro ou fora do modelo hospitalar.

A constatação de que existem teorias de enfermagem capazes de orientar o cuidar e o cuidado para pessoas em sofrimento psíquico nos levou a selecionar uma delas, a teoria de Peplau 4 . Nessa teoria buscamos apreender os conceitos abrangentes capazes de orientar a prática de Enfermagem Psiquiátrica desejada para as novas formas de atenção propostas pelo movimento da Reforma Psiquiátrica Brasileira.

Embora uma definição minuciosa de como deve ser a atuação profissional do enfermeiro não seja explicitada por Peplau ${ }^{4}$, podemos entendê-la como estando baseada em um relacionamento interpessoal, interdisciplinar, comunitário e vinculado à família, o que se aproxima dos conceitos constituintes da Reforma Psiquiátrica no Brasil. Nesse sentido, é importante estabelecer uma conexão entre os pressupostos teóricos de Peplau ${ }^{4}$ e os da Reforma Psiquiátrica, para ter como uma indicação de base conceitual para o cuidar em enfermagem nesse novo paradigma. Mais do que uma indicação, esta conexão poderá subsidiar/ orientar/fundamentar as ações de Enfermagem Psiquiátrica desenvolvidas no hospital e fora dele.

\section{ABORDAGEM METODOLÓGICA}

Este é um estudo de cunho teórico. Diante da complexidade que se configurou na aproximação entre conteúdos bibliográficos distantes, uma teoria americana da década de 50 e princípios para o cuidado em psiquiatria a partir da década de 70 no Brasil, tornou-se imprescindível para um aprofundamento da realidade que se apresentava. Para isso, foi utilizada uma adaptação da análise sistemática através da metanálise para o problema em estudo. A opção por essa forma de olhar sobre os dados fundamentou-se no que afirma Castro3:

A revisão sistemática é uma revisão planejada para responder a uma pergunta específica $e$ que utiliza métodos explícitos e sistemáticos para identificar, selecionar e avaliar criticamente os estudos, e para coletar e analisar os dados destes estudos incluídos na revisão. ${ }^{3: 1}$

Para a elaboração deste estudo, foram utilizados os conceitos da teoria do relacionamento interpessoal apresentados por Peplau4, publicados, em 1952, no seu livro Relação Interpessoal na Enfermagem: um conceito de referência para a enfermagem psicodinâmica. Por conseguinte, a partir do conteúdo das mensagens encontradas nos escritos dessa autora, foi possível estabelecer a utilização de indicadores que permitiram estabelecer associações com a realidade apresentada pelos princípios da Reforma Psiquiátrica Brasileira. 0 objetivo do uso desses indicadores foi o de apreender, primariamente, a abrangência teórica dos conceitos de Peplau para, posteriormente, conduzir um olhar fidedigno sobre as diretrizes da reforma psiquiátrica.

0 tratamento dos resultados foi realizado por meio da categorização e classificação dos elementos constitutivos, definido pela metanálise como análise através de agrupamentos ${ }^{3: 3,6}$.

A revisão sistemática tem por objetivo agrupar os resultados a partir de uma questão específica, porém, esta questão não pode ser tão detalhada. As diferenças encontradas serão uma parte dos resultados. Assim, a formulação de uma questão específica sem critérios rígidos de inclusão e exclusão, variáveis e intervenções estudadas é a forma mais adequada. 0 agrupamento dos resultados é feito de acordo com a qualidade, os participantes e a intervenção ${ }^{3}$.

Dessa maneira, a apreensão dos conceitos apresentados pelo referencial teórico de Hildegard Peplau foram feitos fazendo a identificação dos conceitos aos quais a prática de enfermagem psiquiátrica deveria estar sujeita, tendo como ponto focal a essência dessa teoria: o relacionamento interpessoal. Foram levantados pontos que fossem significativos e levassem a uma reflexão teórica sobre como poderia ser construída uma prática de 
enfermagem psiquiátrica mais flexível e próxima dos conceitos da Reforma Psiquiátrica.

A teoria do relacionamento interpessoal, apresentada por Peplau ${ }^{4}$, deu origem a três categorias, a saber: conhecer a si / enfermeiro; conhecer o outro / paciente; o ambiente, no sentido ampliado, diz respeito a tudo aquilo que circunda e contextualiza 0 indivíduo. Todas elas foram analisadas à luz dos princípios da Reforma Psiquiátrica Brasileira.

Para apreender o conteúdo dos princípios da Reforma Psiquiátrica Brasileira, foram consultados diferentes autores, nomeados como formadores de opinião, para este campo, por Leal ${ }^{5}$ em seu trabalho 0 agente do cuidado na reforma psiquiátrica brasileira.

0 tratamento dos resultados foi realizado através da categorização e classificação dos elementos constitutivos em unidades temáticas. Na teoria do relacionamento interpessoal, apresentada por Peplau ${ }^{4}$, foram delimitados três eixos conceituais: conhecer a si - enfermeiro; conhecer o outro - paciente; e ambiente atual e ambiente de vida.

Tendo em vista a quantidade de autores indicados como formadores de opinião para o campo da Reforma Psiquiátrica Brasileira, os dados foram, primariamente, agrupados em quadros de análise, de modo a permitir uma visualização gráfica, a saber: delimitação da abrangência dos discursos dos formadores de opinião, e suas respectivas publicações; os formadores de opinião, a década das suas publicações e o seus respectivos discursos; e, em última instância, as leituras foram feitas com o intuito de agrupar esses princípios em unidades temáticas - cidadania individual: saberes e práticas clínicas, cidadania coletiva/social: ações coletivas e políticas públicas e habilidade profissional para o exercício da cidadania individual e coletiva.

As conclusões não foram pautadas na análise no poder estatístico, e sim na qualidade dos escritos, a partir das evidências das aproximações e distanciamentos das unidades temáticas da Peplau ${ }^{4}$ e dos princípios da Reforma Psiquiátrica, o que me permitiu evidenciar sua aplicabilidade clínica e condutas padronizadas em estudos de metanálise.

\section{A TEORIA DO RELACIONAMENTO INTERPESSOAL E OS PRINCÍPIOS DA REFORMA PSIQUIÁTRICA BRASILEIRA}

0 processo interpessoal é o ponto focal do livro de Peplau ${ }^{4}$, com seus conceitos voltados para 0 estabelecimento deste tipo de relação. A conceituação em análise é marcada pelo entendimento do relacionamento interpessoal como um processo de aprendizagem. Desse modo, a prática terapêutica deve estar vinculada à necessidade de um conhecimento individual tanto do profissional e cliente, como também da coletividade, para ter aplicabilidade nos contextos de vida das pessoas envolvidas.

Segundo Peplau ${ }^{4}$, três fatores são necessários para a relação interpessoal ser estabelecida como um processo de aprendizagem: o enfermeiro, o paciente e seus respectivos contextos de vida. Esses fatores dão substratos a três eixos conceituais: conhecer a si - enfermeiro; conhecer o outro - paciente; e o entendimento do ambiente, no sentido ampliado que diz respeito a tudo aquilo que circunda e contextualiza o indivíduo.

Entre as posições de Peplau e os princípios da Reforma existe um distanciamento histórico que influencia a utilização de determinadas terminologias. Nos textos sobre a Reforma existe a referência à cidadania. Em Peplau ${ }^{6}$ aparece a necessidade do relacionamento interpessoal ter como meta a manutenção do doente em seu estado de saúde. Nesse sentido, a autora não faz uma exploração conceitual sobre direitos e deveres decorrentes da inserção política e social do indivíduo, que estariam vinculados possivelmente a uma discussão sobre a cidadania alcançável para o doente mental. No entanto, Peplau ${ }^{4}$ parece indicar que a assistência de enfermagem viável é aquela que mantém o paciente na comunidade.

Entendendo que o movimento político da época estava centrado na desospitalização americana, isto é, a retirada dos pacientes dos hospitais psiquiátricos, a autora explora a necessidade da manutenção do estado de saúde do paciente, porém não estando atrelada ao princípio do exercício da cidadania.

Para os autores formadores de opinião, os indícios para a manutenção desse estado de saúde estão atrelados à possibilidade de trabalhar a cidadania do doente mental. Embora explore uma assistência primária, de caráter intervencionista na manutenção do bem-estar do doente, Peplau ${ }^{6}$ não se aprofunda nas questões relativas à cidadania. Não estamos falando de um entrave para a articulação desses conteúdos, mas de nomeações datadas e avanços restritos na época da sua escrita.

Entre Peplau ${ }^{4}$ e os princípios da reforma existe um distanciamento histórico que influencia a utilização de determinadas terminologias. A reforma fala de cidadania e Peplau ${ }^{4}$ da necessidade de o relacionamento interpessoal ter como meta a manutenção do doente em seu estado de saúde.

Diante da existência da necessidade de se restabelecer um estado de saúde, os autores formadores de opinião posicionam-se sobre a maneira 
como deve ser conduzida a assistência psiquiátrica. A cidadania conduz o pensamento sobre algo peculiar em relação às características físicas e psíquicas do paciente. A nomeação individualidade, como conjunto de qualidades que constituem um indivíduo, é delimitadora de capacidades próprias e da possibilidade de criação de suas normas particulares ${ }^{7: 39}$.

A cidadania nos conduz a pensar sobre algo peculiar em relação às características físicas $e$ psíquicas. A nomeação individualidade, enquanto conjunto de qualidades que constituem um indivíduo, é delimitadora de capacidades próprias e da possibilidade de criar suas normas particulares.

Peplau ${ }^{4: 14}$ fala que o problema está em cada paciente ter suas peculiaridades e que, para entendermos melhor com quais particularidades estamos dialogando, é preciso conhecer o ambiente de cada paciente, seu ambiente interpessoal, sua família, sua comunidade 1 .

Os autores formadores de opinião avançam em relação a Peplau ${ }^{4}$ quando centram suas discussões na necessidade de pensar a reabilitação psicossocial desses pacientes como uma atitude estratégica, uma vontade política, uma modalidade compreensiva, complexa e delicada de cuidados para pessoas vulneráveis aos modos de sociabilidade habituais que necessitam de cuidados igualmente complexos e delicados ${ }^{8: 21}$.

Diante do que foi exposto, concluímos que não existe uma técnica de reabilitação mais indicada, mas um conjunto de diferentes técnicas que possam trabalhar com as necessidades individuais e as oportunidades do contexto. A reabilitação não é para adaptar ao jogo dos fortes, os fracos. É um processo para que se mudem as regras e os fortes possam conviver, coexistir, no mesmo cenário que os fracos: ${ }^{9: 151}$.

É possível garantir ao paciente o seu próprio modo de funcionamento por meio do entendimento do conjunto de leis, princípios, costumes e hábitos exclusivos à sua percepção de vida. Nesse sentido, cada um (...) [tem] seu poder de aquisição neste mundo (...) [sendo], às vezes, (...) mais hábeis ou menos hábeis, mais habilitados ou menos habilitados ${ }^{9: 97}$.

Dessa forma, Peplau ${ }^{4}$ explica a necessidade de trabalhar com o entendimento do cliente, visto que indivíduos diferentes reagem diferentemente à doença $a^{4: 18, ~ b . ~ P o r t a n t o, ~ d e v e m o s ~ e s t a r ~ i n t e r e s s a d o s ~ n o ~}$ que leva uma pessoa a procurar um tratamento para enfrentar sua dificuldade de saúde. Somente a partir desse entendimento por parte do enfermeiro é possível que ele pense e implemente seus cuidados de enfermagem. A autora fala de um fator importante no relacionamento interpessoal, o de reconhecer a individualidade do paciente, trabalhar com o problema ou dificuldade apresentado por ele, de modo a poder torná-lo co-participativo no tratamento:22.

Assim, os conteúdos dessa autora e os princípios da Reforma Psiquiátrica convergem para uma necessidade de dar voz ao sujeito. Nos pressupostos da Reforma, é possível entender a clínica como sendo um modo de escutar e agir em função da escuta. Através do entendimento da singularidade do outro, é possível estar autorizado a cuidar e conduzir um tratamento pautado nas capacidades do paciente e na garantia da referência ${ }^{10}$.

$\mathrm{Na}$ construção de sua teoria, Peplau enfatiza a necessidade de escutar o paciente, o que se torna realidade através do emprego do segundo conceito interpessoal: conhecer o outro. Nesse sentido, permitirse escutar é ouvir necessidades. 0 ser humano não possui apenas necessidades concretas de respirar, comer e dormir, mas também necessidades subjetivas e talvez pouco conhecidas e reconhecidas. Viver em grupo e se sentir como parte ativa deste ou daquele grupo, é também uma necessidade que proporciona a obtenção de respostas as mais variadas possíveis. Trabalhando essas respostas pessoais em grupo estabelece-se uma dependência, como um componente necessário nas relações de interdependência das pessoas ${ }^{11: 165}$. 0 trabalho da Enfermagem somente pode ser pensado no sentido de trocas com o indivíduo, com a família, com a comunidade e com outros profissionais. Esta é a essência do que nomeado trabalho co-partícipe.

A estratégia utilizada pela Reforma Psiquiátrica Brasileira é lançar mão de dispositivos comunitários em sua essência, dentro do espaço físico da comunidade e articulado às relações da mesma, para dar maior equilíbrio a esta necessidade política e social do sujeito de pertencer ao espaço em que está inserido. 0 que se busca para a autonomia do doente mental é a dependência de um maior número de trocas. Somos mais autônomos quanto mais dependentes de tantas coisas pudermos ser ${ }^{12: 57}$.

Portanto, quando falamos em autonomia, nossa liberdade moral, necessariamente falamos de dependência, nossa sujeição aos laços sociais que construímos. Entretanto nesta discussão, devemos avaliar em que medida estamos prontos para acolher os doentes psiquiátricos em nossas vidas, para aceitar os estabelecimentos dos centros de atenção psicossocial em nossas vizinhanças 13:13.

Dessa forma, para reunir uma disponibilidade técnica e científica, não basta o profissional estar presente para o paciente. É preciso estar habilitado a 
lidar com esta presença. Esta é a questão na qual se agrupam todos os princípios. Portanto, não adiantaria que novas discussões fossem propostas se não houvesse os meios para implementá-las. 0 que nos conduz a refletir que o início de qualquer trabalho clínico deve estar pautado na seguinte indagação: em que medida profissionais capacitados nos hospícios estarão habilitados ao exercício de uma nova prática? ${ }^{13: 13}$

A difícil tarefa em desconstruir conceitos não se faz apenas no âmbito da sociedade vigente. Talvez, os maiores produtores de um entendimento errôneo sobre a doença mental sejam os próprios profissionais que trabalham com ela. Seu meio de trabalho acaba por perpetuar normas internas, percepções individuais e saberes demarcadores da exclusão social da loucura.

Em adição a esta percepção, Russo ${ }^{14: 17}$ destaca que em:

Cada um de nós, independente da raça, tem um eu primitivo dentro de si, que deve ser educado, civilizado, transformado. Essa transformação, evidentemente, só poderá ser levada a bom termo se guiada por aqueles que iluminados pela nova teoria e despidos dos preconceitos ditados pela tradição, sejam capazes de indicar o bom caminho a ser seguido.

Embora falem do universo simbólico relacionado às construções das nossas identidades, estas citações não parecem indicar uma limitação definitiva para o cuidado, mas um reconhecimento da diferença.

Em se tratando de um campo em transformação, quando falamos em reforma é essencial pensarmos: como podemos reformar algo, de cuja essência ainda não nos aproximamos? Como justificar um posicionamento teórico se não entendemos os motivos de nossas escolhas? Com que verdades estamos dialogando?

A personalidade construída por cada enfermeira determinará a maneira como o contato interpessoal será conduzido em cada situação de enfermagem (Peplau ${ }^{15: 24}$ ). Se a psicanálise assume, como premissa do atendimento, a análise do próprio analista, Peplau alarga esta proposição a todos os enfermeiros sem a obrigação de tornarem-se analistas.

Esta percepção nos conduz a pensar que é necessário ser especial para trabalhar na psiquiatria, no sentido de possuir um saber que é próprio, particular, mas, acima de tudo, ter consciência sobre este saber. Os nossos valores morais, as nossas formas de vida, nos tornam especiais como agentes do cuidado, a partir do momento que os conhecemos e sabemos minimamente como encaminhá-los.

Para esta teórica, a formação de cada enfermeira faz uma diferença substancial no aprendizado sobre a doença vivenciada pelo paciente $e^{4: 67, c}$.
Percebemos que existe uma proximidade entre princípios e conceitos, quando se fala de uma necessidade de conhecer o outro, para prestar ações de cuidado. Com algumas ressalvas, tanto os princípios da Reforma Psiquiátrica Brasileira quanto os conceitos de Peplau articulam-se quando o foco de discussão é conhecer a si para ser possível lidar com o outro.

0 desafio lançado aos profissionais da área de saúde é o de terem uma disponibilidade primária para 0 autoconhecimento e, assim, poderem estar habilitados para lidar com as percepções individuais em prol de um coletivo, seja ele ou a relação interpessoal definida por Peplau 4 , ou o cuidado reformista caracterizado pela Reforma Psiquiátrica.

\section{CONCLUSÃO}

Falar de um referencial teórico elaborado nos anos 1950, cujos conteúdos se aproximam da realidade atual levou à reflexão sobre os motivos pelos quais teoria e sua aplicação prática foram afastando-se com o tempo. Ao longo deste trabalho, concluímos que não é por falta de aplicabilidade.

Cabe discernir sobre esta aplicabilidade e permitir repensar os cuidados atuais a partir de construções anteriores. Fazer com que as nossas ações atuais renovem-se e, ao mesmo tempo, tenham a garantia de um início teórico, um respaldo científico, um fio condutor de novas formas de pensar. Portanto, ainda permanecem questões a serem empiricamente esclarecidas, tais como: 0 que teria acontecido com 0 ensino da enfermagem ao longo dos anos? Por que 0 descaminho ou afastamento? Por que uma teoria com aplicabilidade tão atual foi deixada para trás?

Estudar, com certa modéstia e ciente das limitações apresentadas, os princípios aplicáveis à Reforma faz pensar sobre as diversas dificuldades que surgem quando a proposta é a discussão sobre cidadania e seu exercício no meio social, a reabilitação psicossocial. Cabe ressaltarmos que os distanciamentos, nesta cronologia do tempo e das ações, não permitiu a teórica discutir de uma maneira aprofundada sobre as ações em dispositivos extra-hospitalares e, conseqüentemente, falar das ações clínicas necessárias nestes dispositivos. Peplau não discute sobre reabilitação psicossocial e, neste contexto, não trata do trabalho como meio dinamizador das relações, como fazem os autores formadores de opinião. A teoria do relacionamento interpessoal ensaia uma crítica ao modelo hospitalar e inicia uma discussão sobre a necessidade das ações na comunidade, no meio de vida do sujeito, mas sem aprofundamento.

Desconstruir formas de pensar e agir para produzir novas formas de pensar e agir torna o movimento da 
Reforma especial em sua essência e gerador de desafios constantes. Portanto, seria acertado afirmar que os assuntos pertinentes a uma discussão sobre a Reforma Psiquiátrica Brasileira ainda estão por serem esgotados.

$\mathrm{Na}$ verdade, constituiu-se um desafio analisar um assunto como os princípios da Reforma Psiquiátrica atual, quando ela é pensada, escrita e praticada por diferentes pessoas que, por sua vez, possuem maneiras particulares de perceber o mundo. 0 objetivo que norteia as análises e discussões realizadas pelos formadores de opinião não é único. No entanto, o entendimento da doença mental como experiência humana e seus conseqüentes desdobramentos esbarra a todo o momento com a diversidade de posições apresentada neste campo.

Paralelamente, a vivência experimentada ao longo desta investigação de cunho bibliográfico, que conduziu à formulação do problema estudado, permitiu olhar para além de uma atuação de enfermagem restrita à execução de tarefas. Procuramos demarcar os conceitos que poderiam proporcionar 0 reconhecimento da presença do enfermeiro como fator essencial para a assistência de enfermagem, sob a perspectiva do uso de saberes e fazeres atuais. Essa essencialidade caracterizou-se como uma contribuição para a identidade do enfermeiro psiquiatra.

Como nenhum trabalho que se pretenda científico tem um caráter de terminalidade, ainda permanecem algumas questões que poderão ser exploradas futuramente. Uma delas é a questão da perda do lugar do enfermeiro ao longo dos anos, saindo de uma posição essencial às práticas psiquiátricas hospitalares para se expor a uma certa desvalorização quando os assuntos envolvem centros de atenção psicossocial ou

\section{Referências}

1. Amarante $P$, organizador. Loucos pela vida: a trajetória da reforma psiquiátrica brasileira. Rio de Janeiro (RJ): Ed da FIOCRUZ; 1995.

2. Basaglia F. Considerações sobre uma experiência comunitária. In: Psiquiatria social e reforma psiquiátrica. Rio de Janeiro (RJ): Ed da FIOCRUZ; 1994. p.21-36.

3. Castro AA. Revisão sistemática e meta-análise: Rev Sist Meta-análise. 2001 jun [acesso 11 jun 2004] 1 (1):[aproximadamente 11 telas.] Disponível em: http://www.metodologia.org/meta1.PDF .

4. Peplau HE. Interpersonal relations in nursing. New York (USA): G.P. Putman's; 1991.

5. Leal EM. 0 agente do cuidado na reforma psiquiátrica brasileira: modelos de conhecimento. [tese doutorado em Ciências da Saúde]. Rio de Janeiro(RJ): Instituto de Psiquiatria/ UFRJ; 1999.

6. Peplau HE. Psychotherapeutic strategies. Perspect Psychiatr Care 1968; 35(3);14-9.

7. Goldberg J. Clínica da psicose: um projeto da rede pública. Rio de Janeiro(RJ): Ed Te Corá; 1994. outros dispositivos assistenciais extra-hospitalares. 0 que teria levado o enfermeiro a perder seu lugar para os auxiliares de enfermagem? 0 que faz a diferença no cuidado de enfermagem atual?

0 presente trabalho propôs, ainda, destacar os indícios sobre possíveis caminhos a serem trilhados, sobretudo para a Enfermagem Psiquiátrica, considerando a perspectiva de uma possível teoria a ser contemplada, que não esteja distanciada da realidade prática que se apresenta hoje. Dessa maneira, quando falamos do paciente como portador de uma experiência que lhe é humana, sabemos que ela só existe em termos de contrato social, trocas de dependência, satisfação de necessidades pessoais, de conhecimento do outro - nomeado por Peplau ${ }^{4}$.

Quando refletimos um trabalho comunitário, através dos eixos conceituais propostos por Peplau, sobre é possivel estar nos CAPS, nas Residências Terapêuticas, nos Lares Abrigados, nas casas dos pacientes, na rua, na praça, na igreja, enfim, nos espaços de vida do paciente. Pensamos para além de espaços demarcadores de exclusão, mas produtores de cidadania.

Provavelmente permanecerão indagações ao final desta investigação, já que o trabalho não tem a intenção de esgotar o tema, mas sim de produzir questionamentos sobre práticas relacionadas à assistência em enfermagem psiquiátrica. Podemos considerar que 0 estudo atingiu seu objetivo: produzir indagações direcionadas para uma clínica mais aprimorada para o paciente psiquiátrico, que permita conhecer quem cuidamos, entender seus espaços de circulação e, acima de tudo, entender nossa inserção profissional na produção da dinâmica de uma cidadania possível.

8. PittaA. Reabilitação psicossocial no Brasil. São Paulo(SP): Hucitec; 1996.

9. Saraceno B. Reabilitação psicossocial: uma estratégia para a passagem do milênio. In: Reabilitação psicossocial no Brasil. São Paulo(SP): Hucitec; 1996.

10. Bezerra Junior $B$, Amarante $P$, organizadores). Psiquiatria sem hospício: contribuições ao estudo da reforma psiquiátrica. Rio de Janeiro(RJ): Relume Dumará; 1992.

11. Peplau HE. Peplau's theory of interpersonal relations. Nurs Sci Q. 1997;10 (4):162-67.

12. Kinoshita RT. Contratualidade e reabilitação psicossocial. In: Reabilitação psicossocial no Brasil. São Paulo(RJ): Hucitec; 1996.

13. Silva Filho JF, Leibing AG. A psiquiatria, sua história e seu futuro no Brasil. In: Albuquerque P, Libério M. Práticas ampliadas em saúde mental: desafios e construções do cotidiano. Rio de Janeiro(RJ): IPUB/UFRJ; 1999.

14. Russo JA. A psicanálise enquanto processo civilizador: um projeto para a nação brasileira. Cadernos IPUB. Rio de Janeiro(RJ): Ed UFRJ; 1995.

15. Peplau HE. The psychiatric nurse - accountable? To whom? For what? Perspect Psychiatr Care. 1980; 35(3): 20-5. 


\section{Notas}

aLoucura e doença mental não são a mesma coisa. São termos usados em dois momentos históricos distintos e relacionados à normas sociais, políticas e ideológicas referentes. A loucura assume o estatuto de doença mental, quando ela é percebida como forma de conhecimento humano e é, então, institucionalizada como saber psiquiátrico e médico (Silva Filho et $\mathrm{al}^{13}$ ).

${ }^{\mathrm{b}}$ Texto original: Different individuals react differently to illness. (Peplau $\left.{ }^{4: 18}\right)$. 'Texto Original: The kind of person each nurse becomes makes a substancial difference to what each patient will learn as he is nursed throught his experience with illness (Peplau ${ }^{4: 67}$ )

\section{Sobre as Autoras}

\section{Taís Veronica Macedo Cardoso}

Mestre em Enfermagem pela EEAN/UFRJ. Especialista em enfermagem psiquiátrica pela UNIRIO. Prof ${ }^{\mathrm{a}}$ Assistente da UNIGRANRIO. Membro do Laboratório de Pesquisa em Enfermagem Psiquiátrica - LAPEPS. enftata@hotmail.com

\section{Rosane Mara Pontes de Oliveira}

Profa do Departamento de Enfermagem Médico-Cirúrgica da EEAN/ UFRJ. Mestre em Enfermagem pela EEAN/UFRJ. Doutora em Enfermagem da EEAN/UFRJ. Membro do Laboratório de Pesquisa em Enfermagem Psiquiátrica - LAPEPS.

\section{Cristina Maria Douat Loyola}

Profa Titular da EEAN/ UFRJ. Doutora em Saúde Coletiva. Assessora de Saúde Mental no Ministério da Saúde. Coordenadora do Laboratório de Pesquisa em Enfermagem Psiquiátrica - LAPEPS. 